Miner, K. N., Walker, J. M., Bergman, M. E., Jean, V. A., Cater-Sowell, A., January, S. C., \& Kaunas, C. (2018). From "her" problem to "our" problem: Using an individual lens versus a social-structural lens to understand gender inequity in STEM. Industrial and Organizational Psychology: Perspectives on Science and Practice, 11(2), 267-290.

National Science Foundation, National Center for Science and Engineering Statistics. (2017). Women, Minorities, and Persons with Disabilities in Science and Engineering: 2017. Special Report NSF 17-310. Retrieved from https://www.nsf.gov/statistics/2017/nsf17310/digest/about-this-report/ Smyth, F. L., \& Nosek, B. A. (2015). On the gender-science stereotypes held by scientists: Explicit accord with gender-ratios, implicit accord with scientific identity. Frontiers in Psychology, 6, 415.

\title{
Yes Virginia, There Is a Gender Disparity Problem-and It Goes Beyond STEM
}

\author{
Satoris S. Howes \\ Oregon State University \\ Jaime Henning \\ Eastern Kentucky University \\ Maura J. Mills \\ University of Alabama \\ Ann Hergatt Huffman \\ Northern Arizona University
}

Miner et al. (2018) do an excellent job of bringing the issue of gender disparity within STEM to the forefront of I-O psychology. However, we believe the focus on STEM is woefully inadequate and urge I-O psychologists to think bigger, better, and broader. There are clear problems with the way women are viewed and treated within the workforce, within the United States, and globally. In narrowing the discussion of the problem to target only STEM, we dramatically limit our understanding of and potential impact on the multifaceted and complex gender disparity problem in the world of work. Furthermore, we assert there are additional legitimizing myths that must be addressed in order to yield a more complete picture of the dilemma and allow us to move forward to make an impact.

We are not arguing that I-O psychologists should not care about the gender disparity in STEM. Of course we should. But, as workplace scholars, do we not have a responsibility toward addressing gender disparity in other

Satoris S. Howes, Oregon State University, Cascades Campus; Jaime Henning, Eastern Kentucky University; Maura J. Mills, Department of Management, University of Alabama; Ann Hergatt Huffman, Northern Arizona University.

Correspondence concerning this article should be addressed to Satoris S. Howes, Oregon State University-Cascades, 1500 SW Chandler Ave, Bend, OR 97702. E-mail: satoris.howes@gmail.com 
fields and disciplines as well? There are many non-STEM male-dominated professions (e.g., military, construction laborers, police, carpentry) that are worthy of our attention. In addition, female-dominated occupations are in need of attention as well, particularly considering members of those occupations are often underpaid and undervalued as compared to members of traditionally masculine occupations. Indeed, Miner et al. note that "there has been a tremendous increase in women's labor force participation in the last 50 years ... particularly in the fields of education, health services, retail, and secretarial and administrative assistants" (p. 267). Workers in these fields, however, are often paid considerably less than those in other (less gendered or male-gendered) fields. In a study on occupations with higher rates of women, Levanon, England, and Allison (2009) found that occupations with predominantly female employees had lower pay as compared to occupations with predominantly male employees. Their longitudinal study found evidence of a "devaluation" explanation, that is, it was the gender factor (being female) that negatively affected the pay scale: It was due to the jobs being predominantly female that they were assigned lower pay.

Thus, we can (and should) focus on removing societal barriers to women entering and remaining in STEM fields, but a necessary part of that must also include addressing what is happening in other fields. For instance, why do women predominantly enter into underpaid and/or undervalued fields? Maybe even more importantly, what can be done to combat this devaluation effect? This question is of particular significance given evidence that when women enter a traditionally male-dominated field in greater numbers than men, pay tends to decline, whereas the opposite effect appears to hold true when greater numbers of men enter a traditionally female-dominated field (Levanon et al., 2009). Moreover, research on the so-called "glass escalator effect" suggests that men tend to advance more rapidly within femaledominated fields (e.g., nursing and education), and much like an escalator it involves less effort and is more inevitable compared to women in these fields (Williams, 1992). These findings further support the notion that work performed by women is valued less than the same work performed by men, seemingly regardless of field. In fact, STEM fields may very well be the exception to this rule, with researchers finding that women in some STEM jobs are sometimes paid more than their male counterparts (Busso, 2017). Although these findings do not address the large underrepresentation of women entering into and remaining in STEM fields, they do suggest that once there, women are often valued.

Miner et al. (2018) note that "gendered work practices are especially relevant to understanding women's experiences in male-dominated fields like STEM because the foundation and culture of these fields is framed around the social construction of masculinity and the association between men and 
the public sphere of work" (p. 273). We suggest including a focus on the other side (female-dominated jobs) as a necessary part of the equation in fully understanding and explaining these experiences and inequity from a macro/social-structural perspective in particular.

In much the way that Miner et al. offer three legitimizing myths associated with gender inequity in STEM, we offer four legitimizing myths related to women's (and men's) issues in STEM and other gendered jobs to provide a more complete picture of the issue at hand.

\section{Myth 1: Women Are Underrepresented in All STEM Fields}

Women earn less than one-fifth of computer science, engineering, and physics undergraduate degrees, yet obtain almost an equal number of degrees in biology, chemistry, and mathematics (National Science Foundation, 2014). This does not mean we have the problem half fixed, but it is important to note because it provides an accurate picture of the current landscape. We are doing something right-at least in some areas. We need to figure out what that is, and promulgate those practices throughout the world of work. Importantly, Cheryan, Ziegler, Montoya, and Jiang (2017) argue that we must move beyond examining STEM as one entity. Specifically, they suggest that "disaggregating STEM fields provides an analytical lens through which to evaluate the most likely causes of current underrepresentation" (p. 1). We need to learn what we are doing right in some fields (e.g., biology) and apply these lessons to the most staunchly male dominated fields (e.g., computer science).

Along similar lines, although the evidence does not speak to the issue of underrepresentation, data suggest that in at least two STEM fields (architectural and engineering management, and chemical engineering), women tend to be paid more than their male counterparts (Busso, 2017). This suggests that in some STEM fields, once there, women are valued as much as, if not more than, men $^{1}$. As such, we must examine what we can learn from practices in these fields that may result in more wide-ranging approaches to reducing gender inequity. Furthermore, we must examine what can be done to ensure that when women enter these fields in greater numbers, pay rates do not decrease.

1 This is not to suggest that compensation equates to value or positive treatment in a workplace. Indeed, renowned scientist and Nobel Prize winner Tim Hunt's speech discussing the trouble with "girls" working in science highlighted the issue of mistreatment of and bias against women in science. Nevertheless, pay is an area in which women have been disadvantaged, and therefore moves toward equity are at least a step in the right direction.Furthermore, the backlash Hunt's comment caused on social media, by male and female scientists, demonstrates the support that women have begun to achieve within the sciences. 


\section{Myth 2: Women's Jobs Are Flexible Jobs}

Miner et al. (2018) note that "the desire for a family-flexible career was the strongest predictor of women's diminishing aspirations for STEM careers, higher than demands of the occupation or interest factors" (p. 283). Yet, we question whether these same desires and concerns would not also keep women out of jobs with uniquely intensive labor demands, such as certain fields of law or travel-intense positions. Conversely, healthcare workers, including nurses and home health aides, might have jobs with nonstandard shifts that allow them to work during non-key family hours (e.g., when the family is asleep), yet these shifts have greater negative consequences to health and well-being. Thus, many women may actually work in less flexible jobs, yet pay the price of the flexibility that is required to attend to nonwork demands in terms of health, well-being, and missed opportunities. For example, data suggest that women are over 10 times more likely than men to miss work to care for a sick child and are often not paid for that time off (Ranji \& Salganicoff, 2014), thus missing out on potential earnings. Furthermore, simply offering increased flexibility may not be the answer. Even when that flexibility is available, men are less likely to take advantage of this flexibility in order to attend to family related obligations and are less likely to be punished or "mommy-tracked" for doing so.

I-O psychologists may have much to offer in this area based on our expertise in job design and motivation. Providing opportunities for, and mentoring/training employees on, job crafting may increase the likelihood of attracting and retaining female employees to jobs perceived to be less flexible. That is, rather than seeking "family-friendly" jobs, women should be encouraged to seek the job they want, find a mentor with similar goals, and craft the job in ways that make it appropriately family friendly. Furthermore, the use of job crafting and the resulting flexibility must be encouraged and supported among all employees, not discouraged or penalized for its association with women (or men) attempting to meet family-related needs.

\section{Myth 3: Men Have All the Advantages}

The problem is larger than simply the need for more women to work in STEM jobs. Rather, the problem is that jobs are gendered. We are not going to argue that, as a group, men in the United States are not advantaged in pay and in job prestige-they are. Yet, men may also experience relevant disadvantages - a consideration largely underrecognized. For example, men do not necessarily have the mentoring and encouragement to enter into less traditionally male fields such as nursing and teaching. For many young boys, the statement of "I want to be a nurse" will elicit markedly different responses than will "I want to be a firefighter." For women, the example above would likely evoke a less negative response. Additionally, there are currently many 
(well needed) programs/grant programs that focus on engaging women in fields that are predominantly male. However, the converse is not true.

It is important to consider all individuals, not just women, as we seek equity in the workplace. To the extent that we keep touting the need for women to enter predominantly male fields, we are in some ways diminishing even more the predominantly female fields. We must ensure that our efforts result in forward progress. Are we further separating the genders by focusing on women to the exclusion of men, when we should actually be focusing on countering the notion of gendered jobs in general?

\section{Myth 4: The Solution Is to Ensure Equal Numbers of Men and Women in STEM Jobs}

This is a step to a solution but only a step. As research has shown, as jobs increase in the proportion of men, there is usually an increase in pay, while an increasing proportion of women is usually associated with a decrease in pay. Additionally, just because we reach equality in numbers, it does not mean there will be equality in experience. In much the way that men may be granted an easier pathway to promotions within female-dominated fields (i.e., the glass escalator; Williams, 1992), they may be treated more advantageously in gender-neutral ones as well. There needs to be additional changes made to ensure an end to gender discrimination in these roles more broadly.

For example, Miner et al. (2018) discuss evidence regarding gender stereotypes and notions of the ideal worker, and how these factors likely contribute to gender inequity in male-dominated work contexts. Thus, even if women were equally represented in STEM jobs, these stereotypes and biases will likely continue to have disproportionate effects on the experiences of women in these fields. Therefore, in addition to a focus on recruitment strategies, much work must be done in the area of retaining female workers in STEM and other traditionally male-dominated fields. For example, a focus on creating organizational cultures that value work-life balance and offer family-friendly benefits, in addition to devaluing a focus on an allconsuming devotion to work, may help keep women in these fields while simultaneously enhancing quality of work life for all employees.

\section{Conclusion}

Herein, we have introduced four more myths-myths that need to be dispelled and can be dispelled by I-O psychologists. The focal article authors note that they "encourage I-O psychologists to contribute to early education, STEM training programs, and attraction to STEM careers when they are able to and bring to bear the knowledge we have about implicit biases, climate, and choice" (p. 281). In accordance with the more comprehensive focus that we argue is warranted, we suggest modifying this wording. 
Specifically, we recommend replacing "STEM" with "worthwhile" or "meaningful," and focusing on the value of careers and such factors as personvocation, person-organization, and person-job fit for individuals without mention of age, race, or gender. Although it is true that fewer women choose STEM careers, the careers those women ultimately do choose are still impactful and meaningful and thus should be equally valued not only in accordance with the idea of compensable factors, but in society in general. Indeed, all of the issues raised by the focal article authors-biases, discrimination, and so on-go far beyond STEM.

\title{
References
}

Busso, M. (2017). Women close gender pay gap in two STEM jobs, lag in others. https://www.bloomberg. com/graphics/2017-women-stem-jobs/

Cheryan, S., Ziegler, S. A., Montoya, A. K., \& Jiang, L. (2017). Why are some STEM fields more gender balanced than others? Psychological Bulletin, 143, 1-35. doi:10.1037/bul0000052

Levanon, A., England, P., \& Allison, P. (2009). Occupational feminization and pay: Assessing causal dynamics using 1950-2000 U.S. census data. Social Forces, 88, 865-891.

Miner, K. N., Walker, J. M., Bergman, M. E., Jean, V. A., Carter-Sowell, A., January, S. C., \& Kaunas, C. (2018). From "her" problem to "our" problem: Using an individual lens versus a social-structural lens to understand gender inequity in STEM. Industrial and Organizational Psychology: Perspectives on Science and Practice, 11(2), 267-290.

National Science Foundation. (2014). Integrated postsecondary education data system, 2013, completions survey. National Center for Science and Engineering Statistics: Integrated Science and Engineering Resources Data System (WebCASPAR). Retrieved from https://webcaspar.nsf.gov

Ranji, U., \& Salganicoff, A. (2014). Balancing on shaky ground: Women, work and family health. https://www.kff.org/womens-health-policy/issue-brief/data-note-balancing-on-shakyground-women-work-and-family-health/

Williams, C. (1992). The glass escalator: Hidden advantages for men in "female" professions. Social Problems, 39, 253-267.

\section{Issues of Gender Inequity Go Beyond STEM}

\author{
P. D. Harms and Karen Landay \\ University of Alabama
}

Although Miner et al. (2018) effectively argue that there is a need for greater efforts on the part of I-O psychologists to confront gender inequity in the STEM fields, we feel that the preoccupation with STEM may blind us to other domains where similar issues not only exist but may be even more prevalent and problematic. Specifically, we would argue that more attention needs to be

P. D. Harms, University of Alabama; Karen Landay, University of Alabama.

Correspondence concerning this article should be addressed to P. D. Harms, University of Alabama, 101 Alston Hall, Box 870225, 361 Stadium Drive, Tuscaloosa, AL 35487. E-mail: pdharms@cba.ua.edu 